Available online at www.eccomasproceedia.org

Eccomas Proceedia COMPDYN (2021) 1834-1839

ECCOMAS Proceedia
COMPDYN 2021

$8^{\text {th }}$ ECCOMAS Thematic Conference on Computational Methods in Structural Dynamics and Earthquake Engineering M. Papadrakakis, M. Fragiadakis (eds.) Streamed from Athens, Greece, 28 - 30 June 2021

\title{
A MESOSCALE TENSEGRITY MODEL OF SPIDER DRAGLINE SILK FIBER
}

\author{
A. Amendola ${ }^{1 *}$, N. Sigh ${ }^{1}$, C. Rodenburg ${ }^{2}$, C. Holland ${ }^{2}$, F. Fraternali ${ }^{1}$ \\ ${ }^{1}$ Department of Civil Engineering, University of Salerno, via Giovanni Paolo II, 132, 84084, Fisciano \\ (SA), Italy \\ \{adaamendola1, snarinder, f.fraternali\}@unisa.it \\ ${ }^{2}$ Department of Materials Science \& Engineering, University of Sheffield \\ Sir Robert Hadfield Building, Mappin Street, Sheffield, S1 3JD, UK \\ \{c.rodenburg, christopher.holland\}@sheffield.ac.uk
}

\begin{abstract}
Tensegrity concepts are common in nature and are recognizable, e.g., in every cell, in the microstructure of the spider silk, and in the pattern of bones and tendons for control of locomotion. This work deals with the formulation of a tensegrity model of spider dragline silk fiber at the mesoscale through a multi-domain network approach with tensegrity architecture. The fiber is described as a multi-walled tube formed by coaxial cylindrical networks of beta-sheet crystals (crystalline domains) and polypeptide (amorphous) chains (noncrystalline domains). The given model generalizes a previous one recently appeared in the literature and paves the way to the optimal design of innovative biomimetic fibers with tensegrity architecture.
\end{abstract}

Keywords: Tensegrity, Spider Silk, Multiscale Modeling, Tensile Response. 


\section{INTRODUCTION}

Tensegrity principles are common in nature and exist, e.g., in every cell, in the microstructure of the spider silk, and in the arrangement of bones and tendons for control of locomotion. The present study deals with the formulation of a tensegrity model of spider dragline silk fiber at the mesoscale through a multi-domain network approach [1],[2] with tensegrity architecture [3].

The fiber is described as a multi-walled tube made of coaxial cylindrical networks of b-sheet crystals (crystalline domains) [4] and polypeptide (amorphous) chains (noncrystalline domains) [5]. Each tube is composed of a network of helical-shaped elements, loaded in tension (or strings) [6] and circumferential elements, loaded in compression (or bars).

The strings correspond to domains of amorphous chains attached to crystalline domains at their extremities, while the bars reproduce the compressive stiffening effect that is played by beta-sheet plated crystals in the circumferential direction, when the fiber is longitudinally stretched [3],[6]. Radial links transfer the compressive stresses from one tube to another.

\section{MESOSCALE MECHANICAL MODELING OF A SPIDER SILK FIBER}

We now illustrate the mechanical behavior of the dragline silk fiber at the mesoscopic scale through a multi-domain network approach [1],[2] with tensegrity architecture [3]. The fibre is described as a multi-walled tube made of coaxial cylindrical networks of $\beta$-sheet crystals (crystalline domains) [4] and polypeptide (amorphous) chains (noncrystalline domains, see Fig. 1) [5]. Each tube consists of a network of helical-shaped elements loaded in tension (or strings) [6] and circumferential elements loaded in compression (or bars). The strings are used to model amorphous chains attached to crystalline domains at their extremities, while the bars are introduced to simulate the compressive stiffening effect of the $\beta$-sheet plated crystals in the circumferential direction, when the fibre is stretched along its direction [3],[6]. Radial links reproduce the stresses of compression from one tube to another. The different tubes forming the fibre model describe the homogenised properties of different sections of the core of the fibre. It is indeed largely accepted in the up-to-date literature that the outer lipid, glycol and skin layers minimally contribute to the overall mechanical response of the fibre [6]-[8]. We now focus the attention on the response of the fibre under an isochoric longitudinal stretching deformation, which stretches the fibre up to an engineering strain $\hat{\varepsilon}=(\ell-L) / L$, where $L$ denotes the unstretched length of the fibre, and $\ell$ denotes the stretched length. If we consider that the volume of the fibre remains constant under stretching, as it is written in many experimental studies (refer, e.g., to [9],[10]), the length variation along the longitudinal axis occurs in association with a radial contraction of the fibre. The radial engineering strain $\hat{\varepsilon}_{r}=(r-R) / R$, where $R$ is the radius of the fibre in the unstretched configuration, and $r$ is the stretched radius, is related to the longitudinal strain $\hat{\varepsilon}$ through

$$
\hat{\varepsilon}_{r}=\frac{1}{\sqrt{1+\widehat{\varepsilon}}}-1
$$



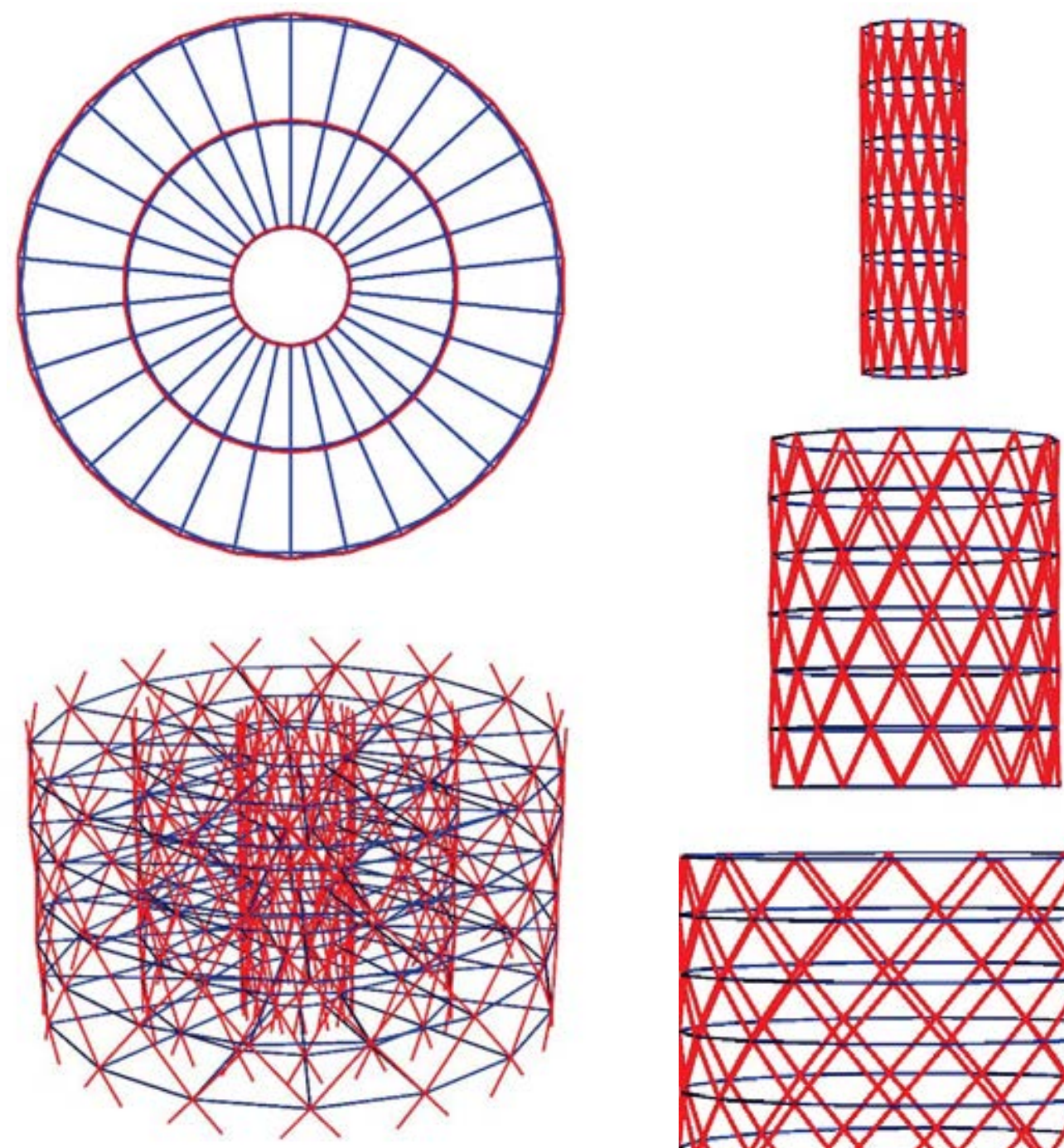

(a)

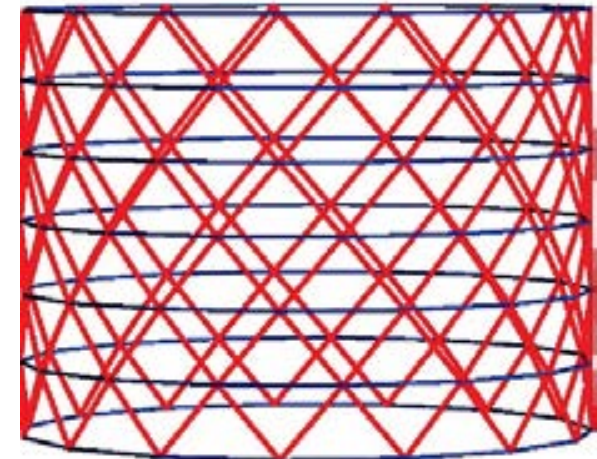

(b)

Fig. 1: Triple-walled tensegrity model of a portion of the dragline silk fiber at the mesoscopic scale: (a) overall views; (b) exploded view of the different walls. The red members are strings or tendons while the blue members are bars or struts.

We characterise the initial elastic response of the strings forming the multiwalled model in Fig. 1 through the following Young modulus:

$$
E_{s}^{(1)}=x E_{a}+(1-x) E_{b}
$$

where $E_{a}$ is the elastic modulus of $\beta$-sheet crystal domains, $E_{b}$ is the initial (pre-yielding) elastic modulus of the noncristalline domanins and $x$ is the volume fraction of $\beta$-sheet crystals within the strings.

The generic string yielding occurs when the engineering strain $\hat{\varepsilon}_{s}$ of the element reaches a threshold value $\hat{\varepsilon}_{s_{y}}$. The mechanical response of the string in the post-yielding regime is described through the following incremental Young modulus: 


$$
E_{s}^{(2)}=x E_{a}+(1-x) E_{c}
$$

where $E_{c}$ denotes the post-yield tangent modulus of the noncrystalline domains.

We consider that the bars are characterized by a constant compressive modulus equal to $E_{a}$, being not affected by yielding phenomena.

Both the strings and the bars of the model (Fig. 1) are considered as rods with rectangular cross section with width $b$ and thickness $t$. By considering the strings of the $i$-th wall, we consider $b=b_{s_{i}}=L_{b_{i}}$, where $L_{b_{i}}$ refers to the initial (undeformed) length of the circumferential bars, and $t$ denotes the radial dimension $t_{i}$ of the layer. Considering the circumferential bars of the $i$-th wall, we assume $b=b_{b_{i}}=s_{c} / 10$ and $t=t_{b_{i}}=t_{s_{i}}$, where $s_{c}$ indicates the longitudinal spacing of such elements. Finally, for what concerns the radial bars we assume $b=b_{r}=L_{b_{3}}$ and $t=t_{r}=s_{c} / 10$. The geometrical and mechanical parameters that describe the proposed model(Fig. 1) are provided in Table 1. Such properties are imported from Refs. [6]-[7] for what concerns the radial dimensions of the layers forming a dragline silk fibre with overall diameter of $5 \mu \mathrm{m}$. The mechanical properties of the fibre elements are instead the following (elastic modulus of the $\beta$-sheet crystals and mechanical properties of the links connecting the crystalline domains, in pre- and post-yielding conditions).

\begin{tabular}{|c|c|c|c|c|c|c|c|c|}
\hline wall & $d(n m)$ & $t(n m)$ & $L_{b}(n m)$ & $x$ & $E_{a}(G P a)$ & $E_{b}(G P a)$ & $\hat{\varepsilon}_{s_{y}}(\%)$ & $E_{c}(M P a)$ \\
\hline 1 & 4100 & 800 & 859 & 0.40 & 20 & 4 & 0.4 & 7 \\
\hline 2 & 2500 & 800 & 524 & 0.40 & 20 & 4 & 0.4 & 7 \\
\hline 3 & 900 & 800 & 189 & 0.40 & 20 & 4 & 0.4 & 7 \\
\hline
\end{tabular}

Table 1. Geometrical and mechanical properties of the model in Fig. 1.

\section{TENSILE RESPONSE}

The use of the properties in Table 1 leads us to predict the engineering and true stressstrain curves illustrated in Fig. 2. The engineering stress $\hat{\sigma}$ is obtained by dividing the total pulling force acting on the fibre by the initial cross section area $\hat{A}$. The true stress $\sigma$ is related to the engineering stress through the equation $\sigma=(1+\hat{\varepsilon}) \hat{\sigma}$, while the true strain $\varepsilon$ is related to the engineering strain through $\varepsilon=\log (1+\hat{\varepsilon})[12]$. 


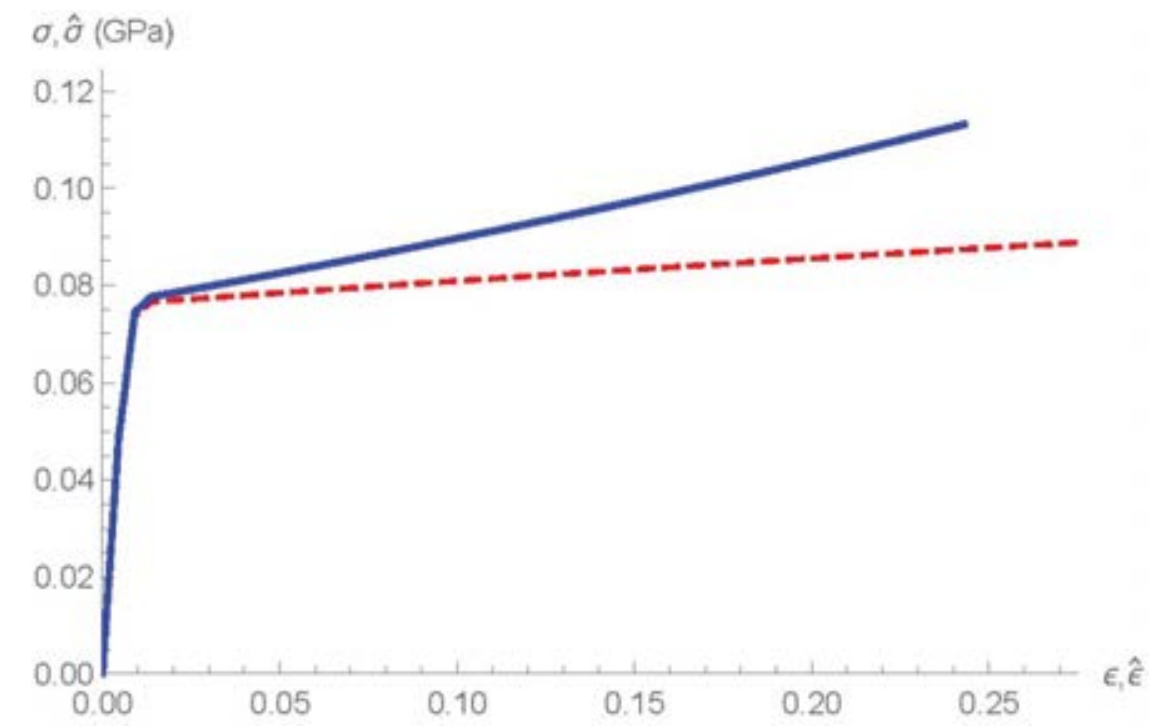

Fig. 2: Engineering (dashed line) and true (solid line) stress-strain responses of the multiwalled silk fibre model.

\section{CONCLUDING REMARKS}

We have illustrated a multi-walled generalization of the tensegrity model recently proposed for the spider dragline silk fiber. The different tubes forming the fiber model describe the homogenized properties of different sections of the core of the fiber. It is indeed largely accepted in the recent literature that the outer lipid, glycol and skin layers contribute very little to the global mechanical behavior of the fiber [6]-[8].

The presented model generalizes the one proposed in [9] and paves the way to the optimal design of innovative biomimetic fibers with tensegrity architecture.

\section{REFERENCES}

[1] Lin N, Liu XY. Correlation between hierarchical structure of crystal networks and macroscopic performance of mesoscopic soft materials and engineering principles. Chem Soc Rev 2015;44(21):7881-915.

[2] Xu G, Gong L, Yang Z, Liu XY. What makes spider silk fibers so strong? from molecular-crystallite network to hierarchical network structures. Soft Matter 2014;10(13):2116-23.

[3] Skelton RE, Nagase K. Tensile tensegrity structures. Int J Space Struct 2012;27(23):131-7.

[4] Keten S, Xu Z, Ihle B, Buehler MJ. Nanoconfinement controls stiffness, strength and mechanical toughness of B-sheet crystals in silk. Nat Mater 2010;9(4):359-67.

[5] Du N, Xiang YL, Narayanan J, Li L, Lim MLM, Li D. Design of superior spider silk: From nanostructure to mechanical properties. Biophys J 2006;91(12):4528-35.

[6] Sponner A, Vater W, Monajembashi S, Unger E, Grosse F, Weisshart K. Composition and hierarchical organisation of a spider silk. PLoS ONE 2007;2(10). 
[7] Stehling N, Abrams KJ, Holland C, Rodenburg C. Revealing spider silk's 3D nanostructure through low temperature plasma etching and advanced low-voltage SEM. Front Mater 2019;5.

[8] Yazawa K, Malay AD, Masunaga H, Numata K. Role of skin layers on mechanical properties and supercontraction of spider dragline silk fiber. Macromol Biosci 2019;19(3).

[9] Fraternali, F., Stehling, N., Amendola, A., Tiban Anrango, B.A., Holland, C., Rodenburg, C. Tensegrity modelling and the high toughness of spider dragline silk. Nanomaterials, 10, 1510, 2020 